\title{
MRI Features for Predicting Microvascular Invasion of Hepatocellular Carcinoma: A Systematic Review and Meta-Analysis
}

\author{
Seung Baek Hong ${ }^{a}$ Sang Hyun Choi ${ }^{b}$ So Yeon Kim ${ }^{b}$ Ju Hyun Shim ${ }^{c}$ \\ Seung Soo Lee ${ }^{b}$ Jae Ho Byun ${ }^{b}$ Seong Ho Park ${ }^{b}$ Kyung Won Kim ${ }^{b}$ Suk Kim ${ }^{a}$ \\ Nam Kyung Lee ${ }^{a}$ \\ aDepartment of Radiology, Biomedical Research Institute, Pusan National University Hospital, Pusan National \\ University School of Medicine, Busan, Republic of Korea; ${ }^{\mathrm{b}}$ Department of Radiology and Research Institute of \\ Radiology, University of Ulsan College of Medicine, Asan Medical Center, Seoul, Republic of Korea; 'Department of \\ Gastroenterology, University of Ulsan College of Medicine, Asan Medical Center, Seoul, Republic of Korea
}

\section{Keywords}

Hepatocellular carcinoma · Magnetic resonance imaging ·

Microvascular invasion · Meta-analysis · Diagnosis

\begin{abstract}
Purpose: Microvascular invasion (MVI) is an important prognostic factor in patients with hepatocellular carcinoma (HCC). However, the reported results of magnetic resonance imaging (MRI) features for predicting MVI of HCC are variable and conflicting. Therefore, this meta-analysis aimed to identify the significant MRI features for MVI of HCC and to determine their diagnostic value. Methods: Original studies reporting the diagnostic performance of MRI for predicting MVI of HCC were identified in MEDLINE and EMBASE up until January 15, 2020. Study quality was assessed using QUADAS-2. A bivariate random-effects model was used to calculate the meta-analytic pooled diagnostic odds ratio (DOR) and $95 \%$ confidence interval $(\mathrm{Cl})$ for each MRI feature for diagnosing MVI in HCC. The meta-analytic pooled sensitivity and specificity were calculated for the significant MRI features. Results: Among 235 screened articles, we found 36 studies including 4,274 HCCs. Of the 15 available MRI features, 7 were significantly associated with MVI: larger tumor size $(>5 \mathrm{~cm})(\mathrm{DOR}=5.2,95 \% \mathrm{Cl}$ [3.0-9.0]), rim arterial en-
\end{abstract}

karger@karger.com www.karger.com/lic

Karger!"

GOPEN ACCESS
(C) 2021 The Author(s)

Published by S. Karger AG, Basel

This article is licensed under the Creative Commons AttributionNonCommercial-NoDerivatives 4.0 International License (CC BYNC-ND) (http://www.karger.com/Services/OpenAccessLicense). Usage and distribution for commercial purposes as well as any distribution of modified material requires written permission. hancement $(4.2,95 \% \mathrm{Cl}[1.7-10.6])$, arterial peritumoral enhancement $(4.4,95 \% \mathrm{Cl}[2.8-6.9])$, peritumoral hypointensity on hepatobiliary phase imaging (HBP) $(8.2,95 \% \mathrm{Cl}[4.4-$ $15.2])$, nonsmooth tumor margin $(3.2,95 \% \mathrm{Cl}[2.2-4.4])$, multifocality $(7.1,95 \% \mathrm{Cl}[2.6-19.5])$, and hypointensity on T1weighted imaging (T1WI) $(4.9,95 \% \mathrm{Cl}$ [2.5-9.6]). Both peritumoral hypointensity on HBP and multifocality showed very high meta-analytic pooled specificities for diagnosing MVI (91.1\% [85.4-94.8\%] and 93.3\% [74.5-98.5\%], respectively). Conclusions: Seven MRI features including larger tumor size, rim arterial enhancement, arterial peritumoral enhancement, peritumoral hypointensity on HBP, nonsmooth margin, multifocality, and hypointensity on T1WI were significant predictors for MVI of HCC. These MRI features predictive of MVI can be useful in the management of HCC.

(c) 2021 The Author(s)

Published by S. Karger AG, Basel

\section{Introduction}

Microvascular invasion (MVI) is an important prognostic factor in patients with hepatocellular carcinoma (HCC) after they have undergone curative treatment such as surgical resection, liver transplantation, or radiofrequency ablation (RFA) and is associated with early tu- 
mor recurrence and poor survival [1-3]. In addition to its use as a prognostic factor, the pretreatment identification of MVI is clinically important for the selection of treatment modalities, that is, surgical resection versus RFA. This is because RFA may be less effective for eradicating intrahepatic metastases and reducing early recurrence rates when HCC shows MVI, with a 90\% 2-year early recurrence rate being reported in patients with a high risk of MVI [2]. However, unlike macrovascular invasion, MVI is difficult to detect on imaging tests such as computed tomography (CT) and magnetic resonance imaging (MRI) and can only be histopathologically diagnosed. Therefore, there is a critical need to determine the pretreatment imaging features for predicting MVI of HCC.

Attempts to detect MVI of HCC using imaging modalities have progressed. Various imaging features on contrast-enhanced ultrasound and CT, including irregular tumor margin or incomplete peritumoral capsule, have been suggested as predictive imaging features for MVI of HCC $[4,5]$. According to the recent advances in MRI, studies reporting predictive imaging features for MVI using MRI are increasing. The use of diffusionweighted imaging (DWI) and hepatobiliary contrast agent (HBA) has given way to the promising results of MRI for detecting MVI of HCC, showing improved diagnostic performance, that is, hypointensity on apparent diffusion coefficient (ADC) was very sensitive (89\%), and peritumoral hypointensity on hepatobiliary phase imaging (HBP) was highly specific (94\%) $[6,7]$.

As clinical interest in MVI of HCC has increased, multiple individual studies have reported various MRI features for predicting MVI of HCC, including a nonsmooth tumor margin [8-12], larger tumor size [11,12], rim arterial enhancement [13], arterial peritumoral enhancement $[2,10,14]$, nonperipheral washout $[15,16]$, nonenhancing capsule [15], diffusion restriction [17-20], tumor hypointensity or peritumoral hypointensity on $\operatorname{HBP}[2,7,8$, $10,21]$, and multifocality $[8,16,22,23]$. However, the reported results seem quite variable, with conflicting reports on which of the MRI features are significant for predicting MVI. Given the clinical importance of MVI in the management of patients with HCC, we considered it timely and important to clearly determine the significant MRI features for predicting MVI. In addition, evaluation of the diagnostic performance of the significant MRI features for predicting MVI can be clinically valuable for understanding the diagnostic characteristics of each imaging feature, that is, which imaging feature is useful for detecting or excluding MVI. Therefore, this meta-analy- sis aimed to identify the significant MRI features for predicting MVI of HCC and to determine their diagnostic value.

\section{Materials and Methods}

This systematic review was performed according to the Preferred Reporting Items for Systematic Reviews and Meta-Analyses (PRISMA) guidelines. This study was prospectively registered in PROSPERO (ID: CRD42020163566).

\section{Literature Search Strategy}

A computerized search of PubMed and EMBASE databases was conducted to identify original studies reporting the diagnostic performance of MRI for predicting MVI of HCC. The search query was designed to furnish a sensitive literature search and included the search terms "Liver," "Carcinoma," "Microvessels," "Invasion," and "MRI." Sources included bibliographic databases, reference lists of eligible studies, review articles, and key journals. The search included articles published up until January 15, 2020, with no start date limits. The studies were restricted to human subjects and those published in the English language. Online suppl. Table 1 (see www.karger.com/doi/10.1159/000513704 for all online suppl. material) lists the search terms in detail.

\section{Eligibility Criteria}

After removal of duplicates, the articles were reviewed to identify studies satisfying the following criteria: (a) population: patients with surgically proven HCC who underwent preoperative MRI; (b) index test: MRI with or without a contrast-enhanced scan; (c) comparison: no comparison; (d) outcome: diagnostic accuracy of MRI findings for diagnosing or predicting MVI in HCC; and (e) study design: any type of study design, including observational studies (retrospective or prospective) and clinical trials. To evaluate the predictive MRI features for MVI of HCC, we did not include studies with macrovascular invasion of HCC on MRI. Studies were excluded according to the following criteria: (a) studies with duplicate patients and data; (b) case reports, review articles, letters, conference abstracts, and editorials; (c) studies with insufficient information to make a diagnostic $2 \times 2$ table for clinical and imaging results; and (d) studies not in the field of interest. The retrieved articles were first screened according to their abstracts and titles, and then full-text reviews of potentially eligible articles were performed. The 2 reviewers (with 8 and 4 years of experience, respectively, in meta-analysis) independently performed the review session, with disagreements being resolved at a consensus meeting with a third reviewer (with 11 years of experience in meta-analysis).

\section{Data Extraction}

The following predefined information was extracted from the eligible articles: (a) study characteristics (authors, years of publication, study period, institution, and study type); (b) subject characteristics (age, the number of patients, the number of HCCs, etiology of HCC, proportion of liver cirrhosis [LC], tumor size, and the type of operation); (c) MRI technique (magnet field strength, the use of contrast-enhanced images, type of contrast agent, and inclusion of DWI); (d) MRI features associated with MVI; (e) outcomes 
(the numbers of true-positives, false-positives, false-negatives, and true-negatives of each MRI feature associated with MVI); (f) reference standard (the surgical method for determining MVI); and (g) image review method (the number of reviewers, consensus or independent review, and clarity of blinding to the reference standard when performing the review).

\section{Assessment of Study Quality}

Two reviewers independently assessed the methodological quality of the eligible articles using the Quality Assessment of Diagnostic Accuracy Studies 2 (QUADAS-2) criteria and its 4 domains of patient selection, index test, reference standard, and flow of patients through the study.

\section{Data Synthesis and Statistical Analysis}

To determine the significant MRI features for diagnosing MVI in HCC, the diagnostic odds ratio (DOR) was adopted as the main study outcome. The DOR is the ratio of the odds of a test being positive if the subject has a disease to the odds of the test being positive if the subject does not have the disease. All MRI features were evaluated, irrespective of their statistical significance, and diagnostic $2 \times 2$ tables were constructed for each individual MRI feature of each study. As a minimum of 4 articles are required to perform meta-analysis, further statistical analysis was performed for each MRI feature that had been reported in at least 4 eligible articles. A bivariate random-effects model was used to calculate the meta-analytic pooled DOR and its $95 \%$ confidence interval (CI) for each individual MRI feature for diagnosing MVI [24]. The meta-analytic pooled sensitivity, specificity, and positive and negative likelihood ratios (LRs) and their 95\% CIs were calculated for each individual MRI feature that was significant for diagnosing MVI according to the meta-analysis. Subgroup analyses according to magnet field strength (3.0-T vs. 1.5-T) and contrast agent (HBA vs. extracellular fluid agent $[\mathrm{ECF}])$ were also performed.

The Higgins $I^{2}$ statistic was used to assess the heterogeneity of the pooled data ( $I^{2}>50 \%$ : substantial heterogeneity) [25]. The presence of a threshold effect was analyzed by visual assessment of the coupled forest plots of sensitivity and specificity, as well as by calculating the Spearman correlation coefficient between sensitivity and the false-positive rate (i.e., 1 - specificity) [26]. A correlation coefficient $>0.6$ was considered to indicate a considerable threshold effect [26]. When substantial heterogeneity was noted, meta-regression analysis was performed to identify the causes. The covariates for the meta-regression were as follows: (a) study design (prospective vs. retrospective); (b) study location (Eastern vs. Western); (c) proportion of LC ( $\geq 50 \%$ vs. $<50 \%, 50 \%$ cutoff value was median value); (d) tumor size (all HCCs $<5 \mathrm{~cm}$ vs. any HCCs $\geq 5 \mathrm{~cm}$ ); (e) MRI magnet field strength (only $3.0-\mathrm{T}$ vs. $1.5-\mathrm{T}$ or both $3-\mathrm{T}$ and $1.5-\mathrm{T}$ ); (f) the use of DWI (used vs. not used or unclear); (g) the type of contrast media (HBA vs. ECF or no contrast agent); (h) prespecified definition of MRI features (clear vs. unclear); (i) image review method (multiple readers with consensus vs. multiple independent or single reviews); (j) clarity for blinding to reference standard (clear vs. unclear); and (k) the type of operation (transplantation vs. resection or both resection and transplantation).

The presence of publication bias was evaluated using Deeks' funnel plot and Deeks' asymmetry test. Stata version 16.0 (StataCorp LP, College Station, TX, USA) was used to perform all statistical analyses, with $p<0.05$ being considered statistically significant.

\section{Results}

\section{Literature Search}

Of the 235 eligible articles, 77 were excluded because of duplication between PubMed and EMBASE. Of the remaining 158 articles, 93 were further excluded after review of their abstracts and titles. During the full-text review, 29 articles were further excluded, leaving 36 studies for inclusion in this study (Fig. 1). Because 1 study had overlapped population with a previous study $[2,10]$, we included the study with a larger population [2].

The characteristics of the individual studies are summarized in Table 1. All 36 studies were cohort studies, with 33 retrospective studies $[2,7-9,11-16,18,20-23$, 27-44] and 3 prospective studies [45-47]. The proportions of LC were variable, with 18 studies having $>50 \%$ of patients with LC $[8,13-15,18,23,28,32-34,36,37,41-$ $43,45-47]$, and 18 studies having $<50 \%$ of patients with LC $[2,7,9,11,12,16,20-22,27,29-31,35,38-40,44]$. Thirteen studies had all HCC size of $<5 \mathrm{~cm}[2,9,12,18$, $21,28,31-34,39,41,43], 23$ studies used HBA contrastenhanced MRI $[2,7-9,11-14,16,20,21,27-31,33,37-$ $41,44]$, and 24 studies used DWI $[8,11,16,18,20,22,23$, $27,28,30-37,39,40,42,44-47]$.

The definitions of the 15 analyzed MRI features are summarized in online suppl. Table 2. Five studies used T1 opposed-phase image for evaluating intralesional fat deposition $[15,16,20,31,34]$, although 1 study was unclear about the use of T1 opposed-phase image [46]. Regarding multifocality, 4 studies used satellite nodules $[8,16,23$, $45]$ and 3 studies used $\geq 2$ lesions [22, 30, 35] as a definition of multifocality. Regarding diffusion restriction, 4 studies were clear about the use of ADC $[16,23,32,39]$, whereas 3 were unclear $[28,34,40]$.

\section{Assessment of the Study Quality according to QUADAS-2}

The overall quality of the included studies is summarized in Figure 2. In the flow and timing domain, 8 studies had a high risk of bias, due to inappropriate interval between the index test and reference standard (over 3 months) $[8,13,15,21,22,45]$ and no inclusion of all patients in the analysis $[8,13,21,22,31,33]$. In the reference standard domain, 1 study had a high risk of bias because it interpreted the reference standard result with the index test result [28]. Twenty-two studies had an unclear risk of bias due to the ambiguity on whether the reference standard result was interpreted without knowledge of the index test result $[2,7-9,12,16,20,21,23,30$, $31,33,37-43$ ] or about how to define pathological MVI
96

Liver Cancer 2021;10:94-106

DOI: $10.1159 / 000513704$
Hong/Choi/Kim/Shim/Lee/Byun/Park/ Kim/Kim/Lee 


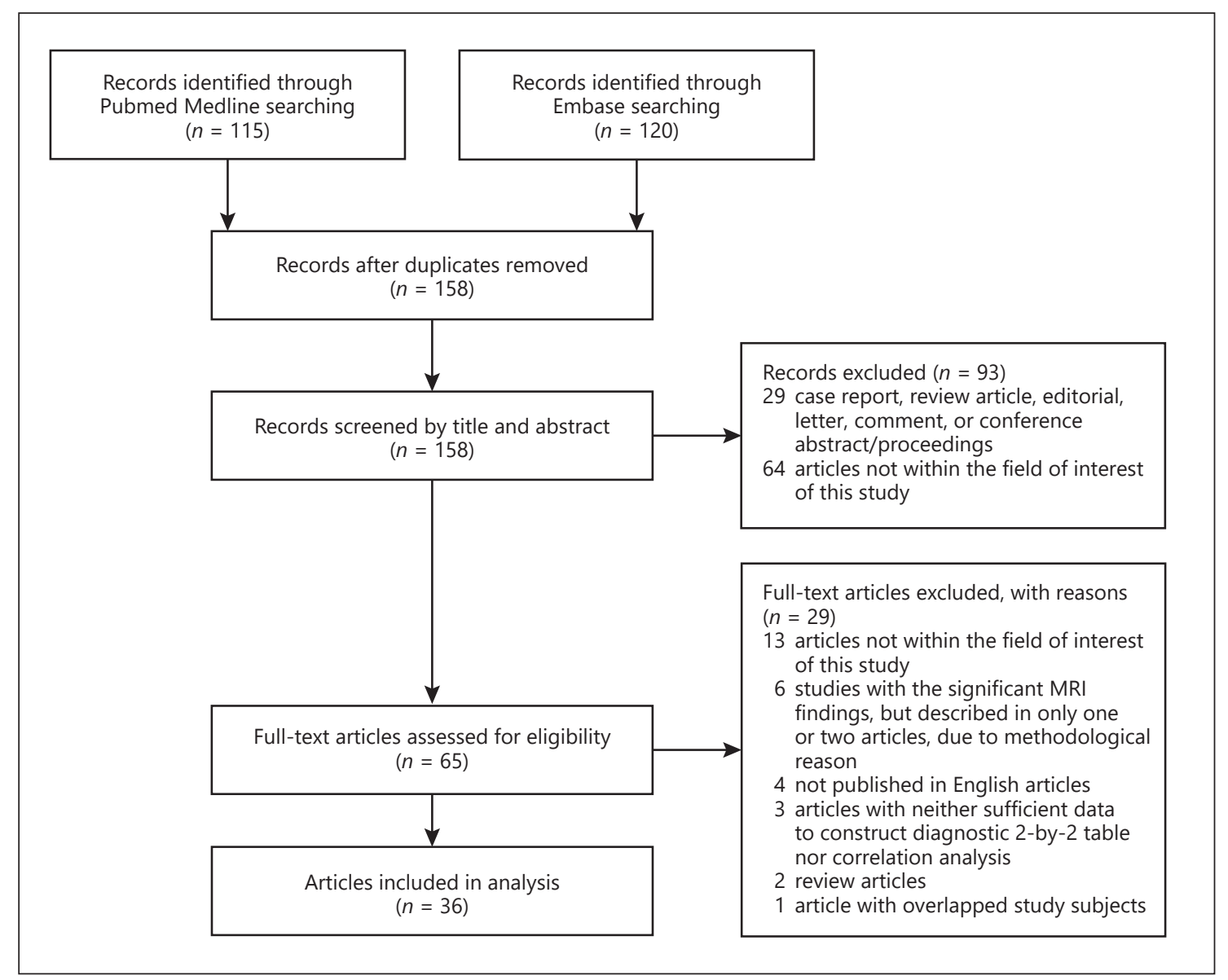

Fig. 1. PRISMA flow diagram of the article selection process.

$[7,16,22,23,27,30,32,33,40]$. In the index test domain, 5 studies were unclear on whether the index test result was interpreted without knowledge of the reference standard $[9,15,29,38,45]$, and 7 studies lacked the prespecified definition of analyzed MRI features $[8,15,22,40$, $42,45,46]$. In the patient selection domains, 6 studies had a high risk of bias due to inappropriate exclusions [22, $31,33,38,41$ ] and case-control design [13]. Seven studies had an unclear risk of bias due to the ambiguity on whether they avoided inappropriate exclusions $[2,7,9$, $11,12,27,35]$.

\section{MRI Features for Diagnosing MVI in HCC}

Of these 36 studies with 4,274 HCCs in 4,039 patients (77\% hepatitis B, 9\% hepatitis C, 3\% alcoholic liver disease, $1 \%$ cryptogenic LC, and 10\% others), 15 MRI features were available for per-lesion based meta-analysis (Table 2). Of these 15 available MRI features, 7 were significantly associated with MVI in HCC (Fig. 3), that is, larger tumor size $(>5 \mathrm{~cm})$, rim arterial enhancement, arterial peritumoral enhancement, peritumoral hypointensity on HBP, nonsmooth tumor margin, multifocality, and hypointensity on T1-weighted imaging (T1WI), demonstrating $95 \%$ CIs of their pooled DORs not enclosing 1.0. Of these 7 significant MRI features, peritumoral hypointensity on HBP showed the highest meta-analytic pooled DOR (8.2, 95\% CI [4.4-15.2]), followed by multifocality $(7.1,95 \%$ CI [2.6-19.5]) and hypointensity on T1WI (4.9, 95\% CI [2.5-9.6]). Diffusion restriction did not show a statistically significant association with MVI of $\operatorname{HCC}(3.6,95 \%$ CI [0.3-35.8]).

All 7 of the significant MRI features were also significantly associated with MVI of HCC in both 3.0-T and 1.5-T MRI groups, and both HBA- and ECF-MRI, except for rim arterial hyperenhancement in ECF-MRI, which showed borderline significance (online suppl. Table 2). Although diffusion restriction was not significantly associated with MVI of HCC on 3.0-T MRI, HBA-MRI, and 


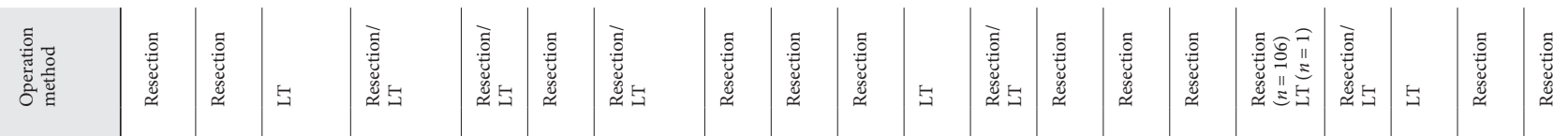

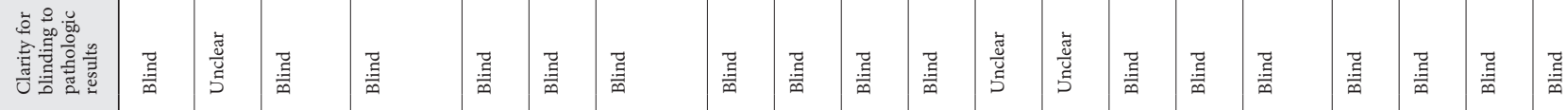
In

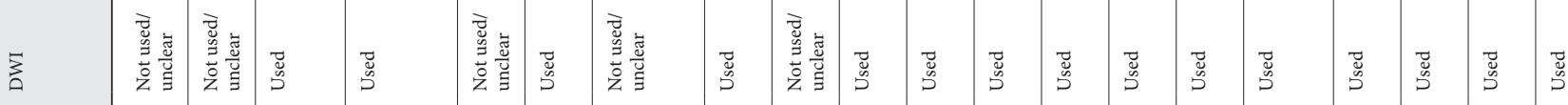

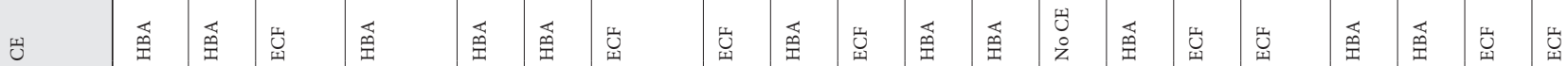

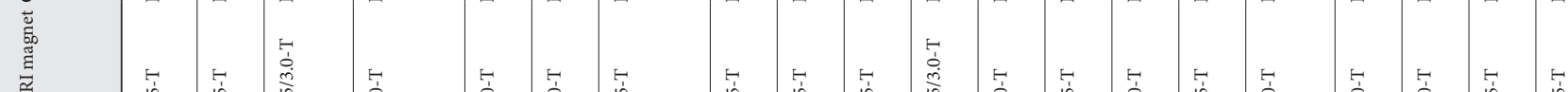

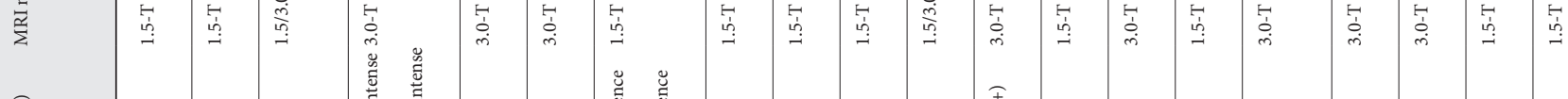

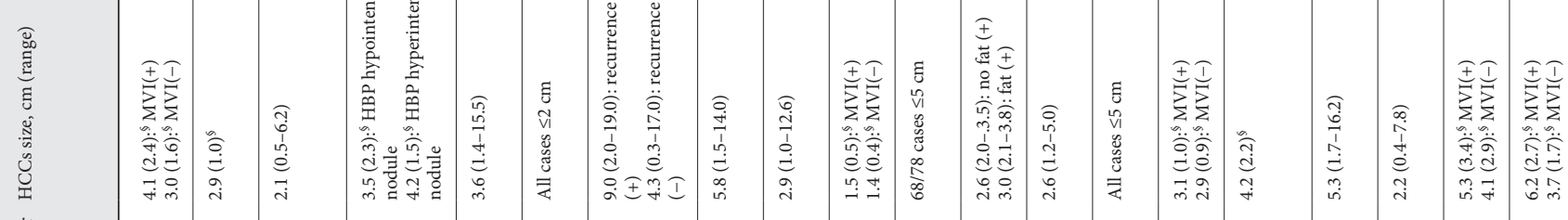

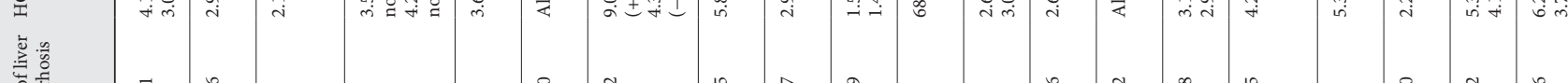

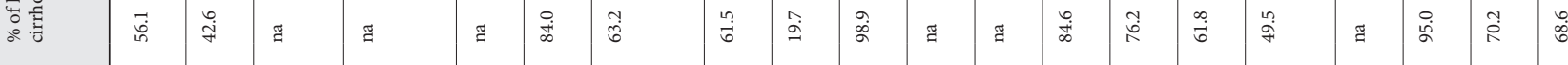

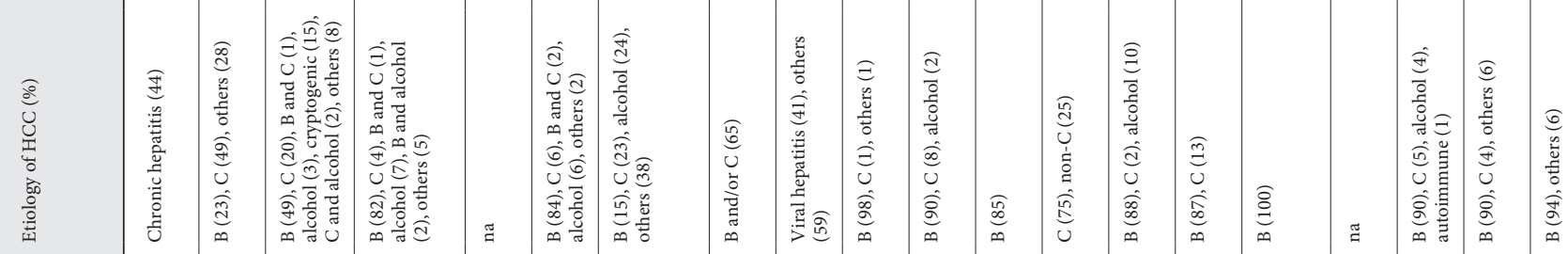
(2)

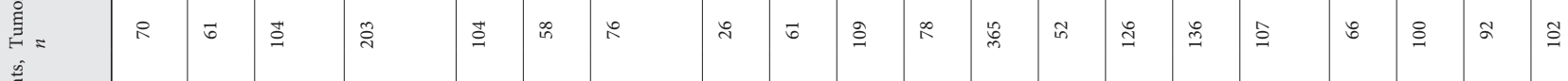

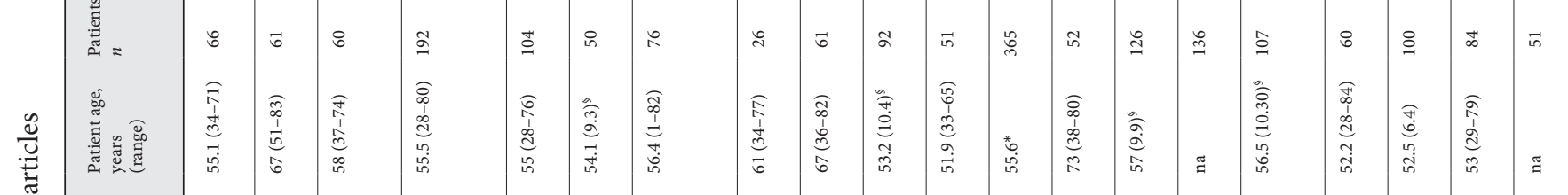

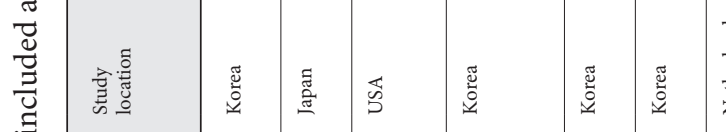

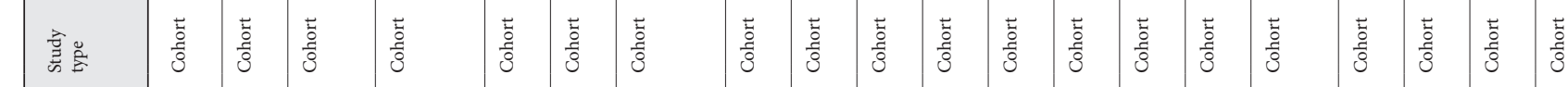

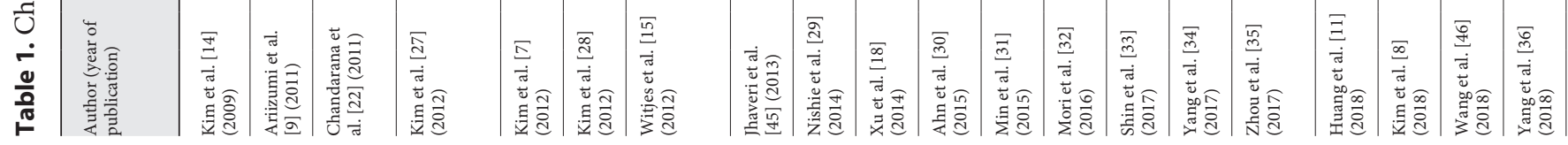




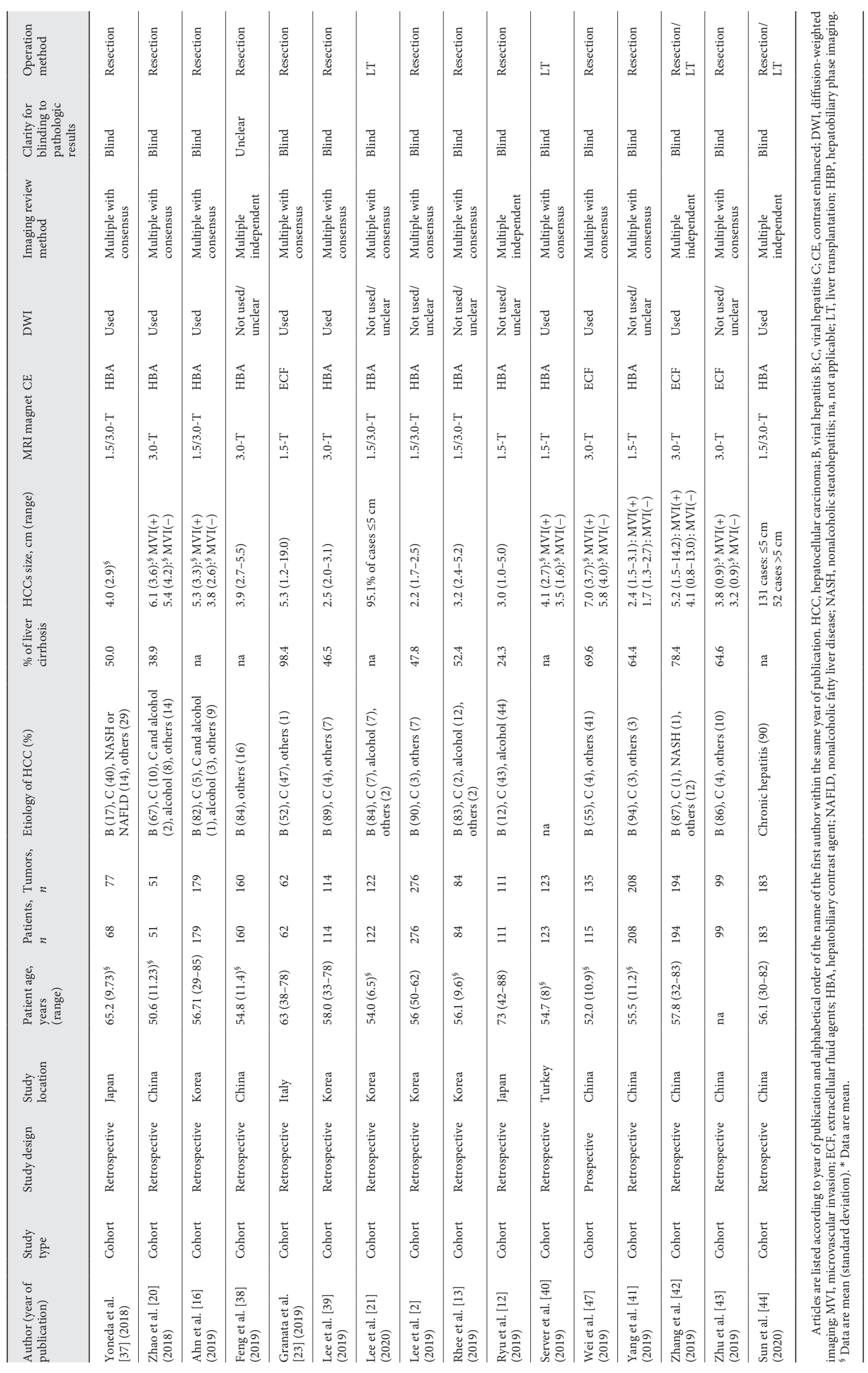




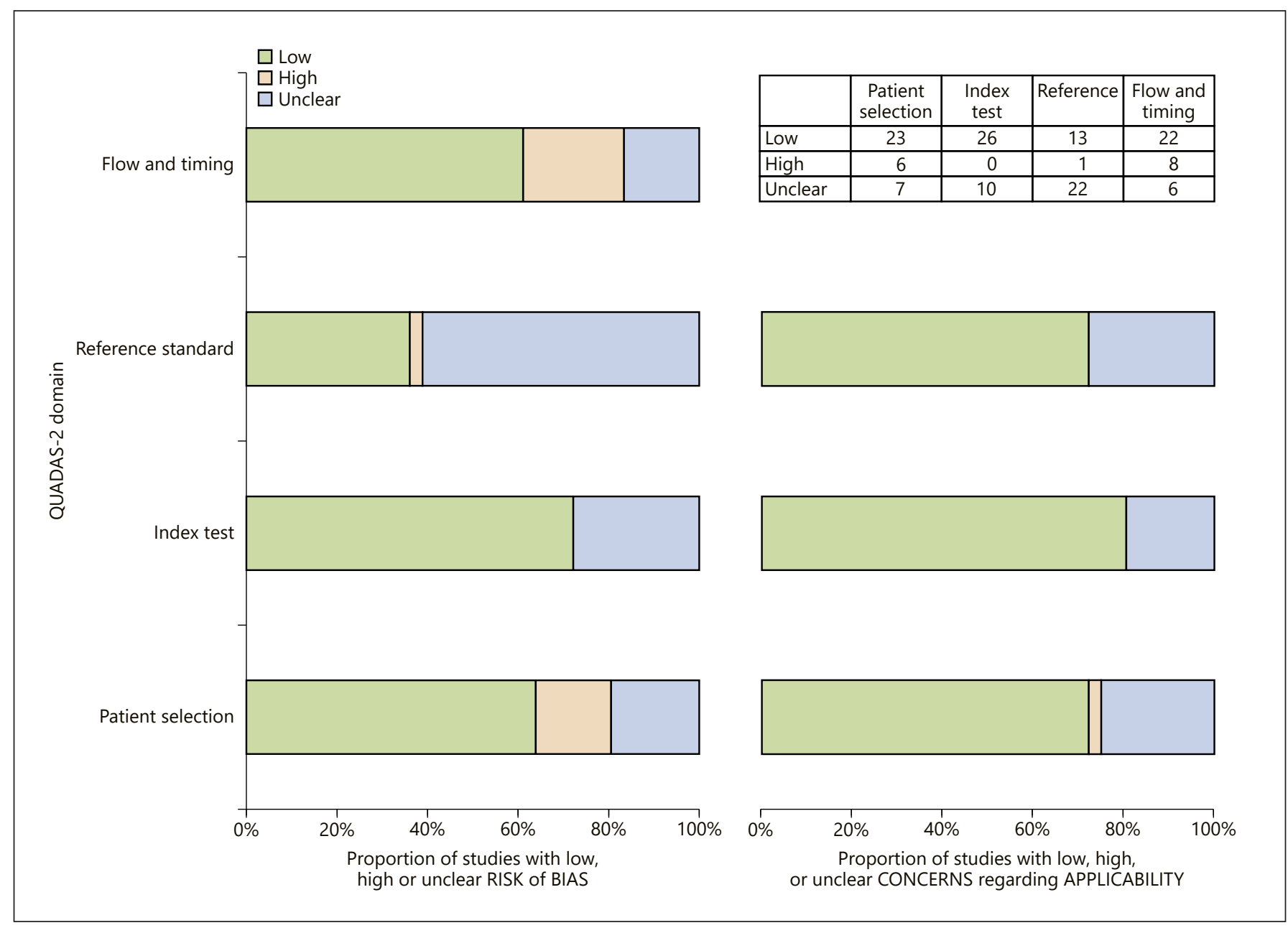

Fig. 2. Quality assessments of the articles according to QUADAS-2 criteria. The methodological quality of the articles is presented as the proportion of articles (0-100\%) with low (i.e., high quality), high, or unclear risks of bias and the proportion of articles with low (i.e., high quality), high, or unclear concerns regarding applicability for each domain.

ECF-MRI, it was significantly associated with MVI of HCC on 1.5-T MRI.

The meta-analytic pooled sensitivities and specificities of these 7 MRI features are summarized in Table 3 (online suppl. Fig. 1). Of these 7 MRI features, hypointensity on T1WI showed the highest sensitivity $(89.4 \%, 95 \%$ CI [72.5-96.4\%]), followed by a nonsmooth tumor margin (67.1\%, 95\% CI [57.7-75.3\%]). In contrast, multifocality showed the highest specificity $(93.3 \%$, 95\% CI [74.598.5\%]), followed by peritumoral hypointensity on HBP (91.1\%, 95\% CI [85.4-94.8\%]). The positive and negative LRs of the imaging findings ranged from 1.4 to 5.0 and 0.3 to 0.7 , respectively.

Substantial study heterogeneity was noted for all 7 significant MRI features $\left(I^{2} \geq 70.4 \%\right)$, but there was no sig- nificant publication bias ( $p \geq 0.16$; online suppl. Fig. 2). Of the 7 MRI features, there was a significant threshold effect in larger tumor size $(>5 \mathrm{~cm})$, arterial peritumoral enhancement, multifocality, and hypointensity on T1WI (Spearman correlation coefficient $\geq 0.8$ ). Nonsmooth tumor margin showed a borderline threshold effect (Spearman correlation coefficient $=0.52$ ).

\section{Meta-Regression Analysis}

The results of the meta-regression analysis are summarized in online suppl. Table 4 . Of the 11 covariates, 2 factors including study location $(p=0.01)$ and proportion of LC ( $p=0.04)$ were significantly associated with study heterogeneity. Eastern studies showed a higher specificity (91 vs. 66\%) in rim arterial enhancement than Western 
Table 2. Summary of the meta-analytic pooled DOR for the imaging findings

\begin{tabular}{|c|c|c|c|c|c|}
\hline \multirow[t]{2}{*}{ MRI features } & \multirow{2}{*}{$\begin{array}{l}\text { Studies, } \\
n\end{array}$} & \multirow{2}{*}{$\begin{array}{l}\text { Cases, } \\
n\end{array}$} & \multicolumn{2}{|c|}{ Summary estimate } & \multirow{2}{*}{$\begin{array}{l}p \text { for } \\
\text { publication } \\
\text { bias }\end{array}$} \\
\hline & & & $\begin{array}{l}\text { pooled DOR } \\
(95 \% \mathrm{CI})\end{array}$ & $I^{2}, \%^{\dagger}$ & \\
\hline Larger tumor size $(>5 \mathrm{~cm})^{*}$ & 4 & 472 & $5.2(3.0-9.0)$ & 70.4 & 0.16 \\
\hline Arterial hyperenhancement & 7 & 493 & $1.0(0.5-1.8)$ & 74.4 & 0.49 \\
\hline Rim arterial enhancement* & 4 & 616 & $4.2(1.7-10.6)$ & 95.5 & 0.93 \\
\hline Arterial peritumoral enhancement* & 12 & 1,510 & $4.4(2.8-6.9)$ & 100.0 & 0.66 \\
\hline Enhancing capsule & 16 & 1,735 & $0.9(0.6-1.3)$ & 99.8 & 0.78 \\
\hline Washout on portal venous phase & 4 & 418 & $1.7(0.9-3.1)$ & 82.0 & 0.05 \\
\hline Washout on delayed phase & 6 & 531 & $1.7(0.9-3.0)$ & 93.1 & 0.35 \\
\hline Hypointensity on HBP & 6 & 993 & $2.1(1.0-4.3)$ & 89.1 & 0.30 \\
\hline Peritumoral hypointensity on $\mathrm{HBP}^{*}$ & 11 & 1,429 & $8.2(4.4-15.2)$ & 100.0 & 0.72 \\
\hline Nonsmooth tumor margin* & 17 & 1,916 & $3.2(2.2-4.4)$ & 99.8 & 0.78 \\
\hline No fat deposition & 6 & 899 & $1.3(0.9-1.9)$ & 64.5 & 0.39 \\
\hline Multifocality* & 7 & 612 & $7.1(2.6-19.5)$ & 98.7 & 0.80 \\
\hline Hypointensity on T1WI* & 5 & 450 & $4.9(2.5-9.6)$ & 73.1 & 0.97 \\
\hline Hyperintensity on T2WI & 4 & 348 & $2.9(0.9-9.0)$ & 27.0 & 0.67 \\
\hline Diffusion restriction & 7 & 724 & $3.6(0.3-35.8)$ & 90.5 & 0.10 \\
\hline
\end{tabular}

DOR, diagnostic odds ratio; HBP, hepatobiliary phase imaging; T1WI, T1-weighted imaging; T2WI, T2weighted imaging. * These imaging findings demonstrated statistically significant meta-analytic pooled DOR. ${ }^{\dagger} I^{2}$ is the Higgin index for heterogeneity ( $>50 \%$ represent substantial heterogeneity).

studies. Studies with $>50 \%$ patients with LC showed a lower sensitivity in nonsmooth margin (57 vs. $77 \%$ ) than studies with $\leq 50 \%$ patients with LC.

\section{Discussion}

This meta-analysis provided a comprehensive summary for MRI features for predicting MVI of HCC including 36 studies with 4,274 HCCs. We found 7 significant MRI features for predicting MVI, that is, larger tumor size $(>5 \mathrm{~cm})$, rim arterial enhancement, arterial peritumoral enhancement, peritumoral hypointensity on HBP, nonsmooth tumor margin, multifocality, and hypointensity on T1WI. This result would be useful in the pretreatment prediction of MVI and facilitate more evidence-based risk stratification in the management of HCC.

In accordance with the study by Lee et al. [10], arterial peritumoral enhancement, nonsmooth tumor margin, and peritumoral hypointensity on HBP were common significant MRI features. However, our meta-analysis identified the 4 additional features including larger tumor size $(>5 \mathrm{~cm})$, rim arterial enhancement, multifocality, and hypointensity on T1WI. The number of subjects and an- alyzed MRI features in previous individual studies was generally insufficient, with $91.7 \%(33 / 36)$ of studies including $<200$ patients $[7-9,11-16,18,20-23,27-30,32-$ $40,42-47]$ and $94.4 \%(34 / 36)$ analyzing $<10$ imaging features $[2,7-9,11-15,18,20-23,27-29,31-47]$. Furthermore, the analyzed MRI features varied across the previous studies, with the selection criteria used for the MRI features being unclear, and the individual studies might therefore have been limited in their ability to determine significant MRI features for predicting MVI. In contrast, given the large number of subjects $(4,410$ patients) and MRI features analyzed (15 features) in this meta-analysis, our results should be beneficial for making more solid conclusions on the MRI features for predicting MVI.

Peritumoral hypointensity on HBP was the MRI feature most suggestive of MVI. Because the meta-analytic DOR and pooled positive LR of peritumoral hypointensity on HBP were 8.2 and 5.0, respectively, this imaging feature can be useful for ruling in MVI [48]. The association of peritumoral hypointensity on HBP with MVI could be explained by peritumoral perfusion change resulting from the dysfunction of organic anion-transporting polypeptide transporters in the hepatocytes around the HCC [10]. Likewise, arterial peritumoral enhance- 


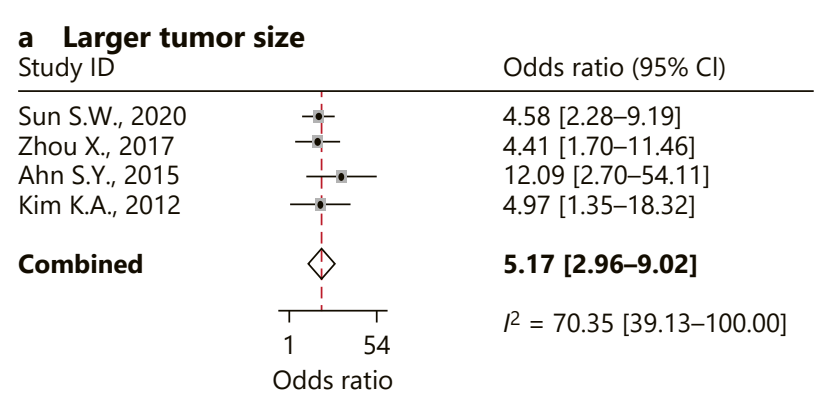

c Arterial peritumoral enhancement Study ID

\begin{tabular}{|c|c|c|}
\hline Zhang R., 2019 & $\frac{1}{\perp} \bullet-$ & $18.60[1.04-331.86]$ \\
\hline Wei Y., 2019 & - & $4.73[2.10-10.67]$ \\
\hline Kim H., 2009 & $-6-$ & $6.00[2.06-17.48]$ \\
\hline Yang L., 2019 & 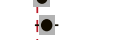 & $8.55[3.63-20.14]$ \\
\hline Lee S. (3), 2019 & $\bullet$ & $11.96[6.45-22.20]$ \\
\hline Ahn S.J., 2019 & - 1 & $1.98[1.06-3.71]$ \\
\hline Feng S.T., 2019 & $\bullet$ & $1.67[0.83-3.38]$ \\
\hline Ahn S.Y., 2015 & $-\bullet-$ & $7.00[1.71-28.68]$ \\
\hline Zhou X., 2017 & $-\bullet$ & $1.52[0.58-3.96]$ \\
\hline Xu P., 2014 & $\because$ & $4.95[1.41-17.35]$ \\
\hline Wang W.T., 2018 & $-0-$ & $6.25[1.25-31.32]$ \\
\hline Kim A.Y., 2018 & $-\dot{0}-$ & $3.66[0.74-18.19]$ \\
\hline \multirow[t]{4}{*}{ Combined } & $\diamond$ & $4.35[2.75-6.88]$ \\
\hline & & $R=100.00[100.00-100.00]$ \\
\hline & 1332 & $x^{2}=100.00[100.00-100.00]$ \\
\hline & Odds ratio & \\
\hline
\end{tabular}

e Non-smooth tumor margin

\begin{tabular}{|c|c|c|}
\hline Study ID & $y$ & Odds ratio $(95 \% \mathrm{Cl})$ \\
\hline Ariizumi S.I., 2011 & ᄂ.- & $25.71[3.01-219.94]$ \\
\hline Zhou X., 2017 & $\frac{1}{7--}$ & $6.38[2.02-20.13]$ \\
\hline Lee S. (3), 2019 & i. & 7.34 [3.85-13.99] \\
\hline Yang L., 2019 & - & $7.22[2.88-18.07]$ \\
\hline Feng S.T., 2019 & .0 & $1.66[0.79-3.51]$ \\
\hline Chandarana H., 2011 & $-\bullet-1$ & $0.57[0.15-2.14]$ \\
\hline Kim A.Y., 2018 & $!$ & $5.32[2.24-12.65]$ \\
\hline Ryu T., 2019 & to. & $5.70[2.51-12.96]$ \\
\hline Wang W.T., 2018 & -6 & $2.33[0.98-5.56]$ \\
\hline Ahn S.J., 2019 & i & $2.95[1.57-5.56]$ \\
\hline Wei Y., 2019 & 0 & $1.55[0.76-3.15]$ \\
\hline Ahn S.Y., 2015 & $-\frac{1}{1-2}$ & $3.14[1.06-9.34]$ \\
\hline Jhaveri K.S., 2013 & -0 & $2.63[0.53-13.07]$ \\
\hline Yang C., 2017 & 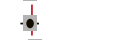 & $2.61[1.25-5.46]$ \\
\hline Kim H., 2009 & $-0-$ & $4.01[1.43-11.25]$ \\
\hline Witjes C.D.M., 2012 & -1 & $1.59[0.46-5.51]$ \\
\hline Xu P., 2014 & -1 & $1.25[0.46-3.37]$ \\
\hline Combined & $\diamond$ & $3.15[2.23-4.44]$ \\
\hline & 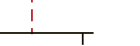 & \\
\hline & 220 & $k^{2}=99.19[199.17-99.81]$ \\
\hline & ds ratio & \\
\hline
\end{tabular}

b Rim arterial enhancement

\begin{tabular}{|c|c|c|}
\hline \multicolumn{2}{|c|}{ Study ID } & $\begin{array}{l}\text { Odds ratio }(95 \% \mathrm{Cl}) \\
18.18[3.88-85.14]\end{array}$ \\
\hline $\begin{array}{l}\text { Rhee H., } 2019 \\
\text { Granata V., } 2019 \\
\text { Lee S. (3), } 2019 \\
\text { Zhang R., } 2019\end{array}$ & $\underbrace{-1}_{-\infty}-\infty$ & $\begin{array}{l}18.18[3.88-85.14] \\
1.55[0.50-4.74] \\
5.96[3.02-11.76] \\
3.00[1.39-6.45]\end{array}$ \\
\hline \multirow[t]{2}{*}{ Combined } & 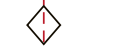 & $4.18[1.65-10.59]$ \\
\hline & $\begin{array}{lc} & 1 \\
1 & 85 \\
\text { Ddds ratio }\end{array}$ & $R=95.47[92.51-98.43]$ \\
\hline
\end{tabular}

d Peritumoral hypointensity on HBP

\begin{tabular}{|c|c|c|}
\hline Study ID & & Odds ratio $(95 \% \mathrm{Cl})$ \\
\hline Lee S. (2), 2020 & $-\bullet-$ & 61.44 [15.96-236.49] \\
\hline Nishie A., 2014 & - & $10.65[3.20-35.42]$ \\
\hline Lee S. (3), 2019 & $\bullet$ & $21.03[10.34-42.75]$ \\
\hline Yoneda N., 2018 & & $6.07[2.13-17.28]$ \\
\hline Ahn S.J., 2019 & - & $3.47[1.81-6.62]$ \\
\hline Shin S.K., 2017 & & $22.12[5.64-86.74]$ \\
\hline Kim K.A., 2012 & & $8.50[2.36-30.63]$ \\
\hline Feng S.T., 2019 & 01 & $1.52[0.77-3.03]$ \\
\hline Yang L., 2019 & -0 & $4.25[1.59-11.36]$ \\
\hline Kim A.Y., 2018 & & 13.62 [1.71-108.73] \\
\hline Ahn S.Y., 2015 & & $3.63[0.47-27.78]$ \\
\hline \multirow[t]{3}{*}{ Combined } & 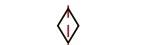 & $8.16[4.37-15.23]$ \\
\hline & $\begin{array}{c}T \\
1\end{array}$ & $R^{2}=100.00[100.00-100.00]$ \\
\hline & Odds ratio & \\
\hline
\end{tabular}

f Multifocality

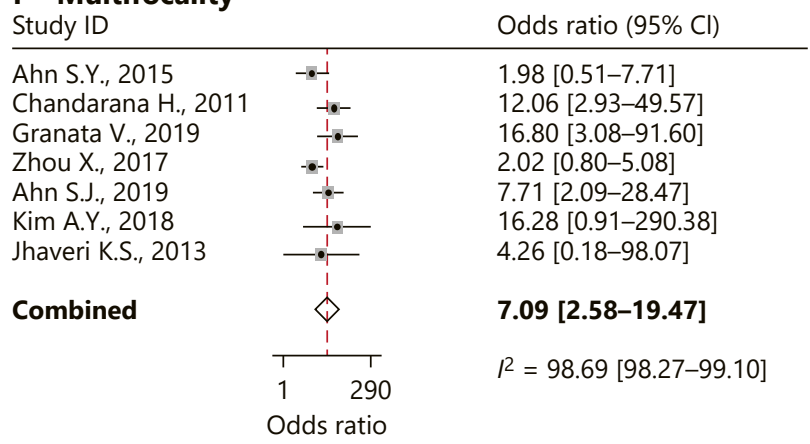

g Hypointensity on T1WI

\begin{tabular}{|c|c|c|}
\hline \multicolumn{2}{|c|}{ Study ID } & \multirow{2}{*}{$\begin{array}{l}\text { Odds ratio }(95 \% \mathrm{Cl}) \\
5.24[0.65-42.33]\end{array}$} \\
\hline Yang C., 2017 & $-\bullet$ & \\
\hline Yang C., 2018 & $\underset{-\bullet-}{T}-$ & $7.73[1.62-36.95]$ \\
\hline Kim M.J., 2010 & -1 & $13.36[1.61-110.78]$ \\
\hline Witjes C.D.M., 2012 & $-\bullet-$ & $2.44[0.78-7.63]$ \\
\hline Ahn S.Y., 2015 & - & $4.32[1.43-13.07]$ \\
\hline \multirow[t]{3}{*}{ Combined } & 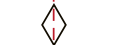 & $4.92[2.51-9.64]$ \\
\hline & $\begin{array}{ll}T \\
1 & 111\end{array}$ & $R^{2}=73.09[48.43-97.76]$ \\
\hline & Odds ratio & \\
\hline
\end{tabular}

Fig. 3. Forest plots of the DORs of larger tumor size $(>5 \mathrm{~cm})(\mathbf{a})$, rim arterial enhancement (b), arterial peritumoral enhancement (c), peritumoral hypointensity on HBP (d), nonsmooth tumor margin (e), multifocality (f), and hypointensity on T1WI (g). DORs, diagnostic odds ratio; HBP, hepatobiliary phase imaging; T1WI, T1weighted imaging. 


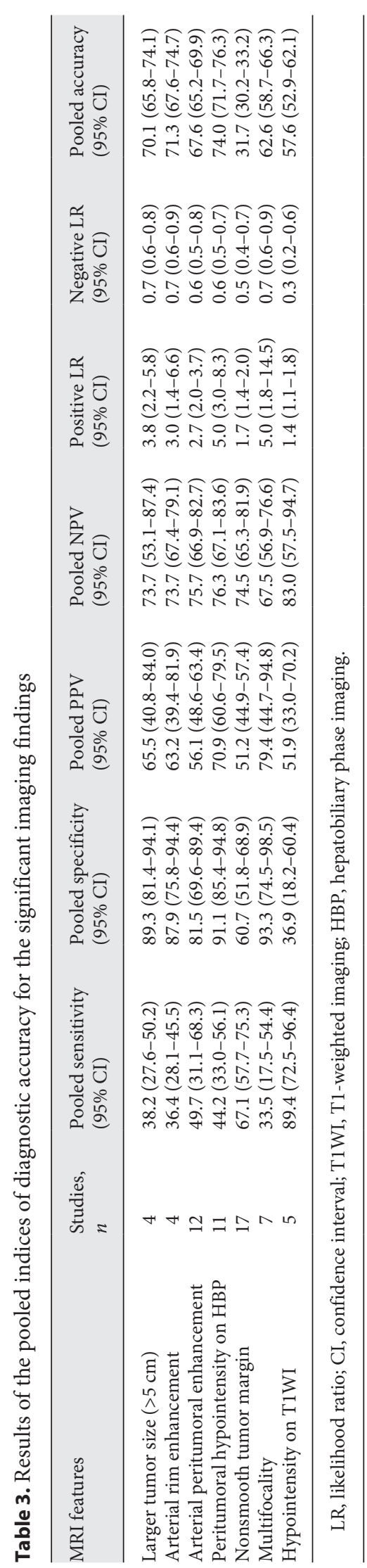

MRI Features for Predicting MVI of HCC ment might be associated with perfusion changes following MVI of HCC. Several studies suggested that arterial peritumoral hyperenhancement could be due to arterial hyperperfusion compensating for decreased portal flow, with this decreased portal flow being caused by microscopic tumor thrombi around the tumor obstructing the minute portal vein branches $[49,50]$.

Multifocality was an MRI feature strongly suggestive of MVI. The significant association between multifocality on MRI and MVI would be explained by the fact that intrahepatic metastases are derived from the main tumor via the portal vein [51]. Because satellite nodules are associated with early recurrence and high tumor grade, as well as MVI [16], the detection of satellite nodules on MRI is important in the management of patients with HCC. In addition, a nonsmooth tumor margin was also an important imaging finding for predicting MVI, with a moderate sensitivity and specificity. This result is consistent with a previous study that reported that the single nodular with extranodular growth-type HCC and confluent multinodular-type HCC correspond with a nonsmooth tumor margin and have a higher risk of MVI than the single nodular type [52]. Although rim arterial enhancement is not a typical MRI feature of HCC, HCC showing rim arterial enhancement can be clinically important because it is associated with MVI, rapid prognosis, and early recurrence [53]. Notably, HCC showing irregular rim arterial enhancement and less enhancement of the central portion of the tumor is associated with low microvascular density and a sinusoid-like pattern, which are features associated with a higher frequency of MVI [13]. In addition, hypointensity on T1WI was one of the significant MRI features for predicting MVI of HCC in this study. Considering the facts that tumor grade was highly correlated with MVI (12\% of well-differentiated HCC had MVI, but 50\% of poorly differentiated HCC had MVI), and that HCC with hypointensity on T1WI tends to be more poorly differentiated $[54,55]$, hypointensity on T1WI would be reasonable as a factor for predicting MVI.

Although there were conflicting reported data whether tumor size was significantly associated with MVI [ 7,15 , $30,56,57]$, this meta-analysis found that a larger tumor size $(>5 \mathrm{~cm})$ was one of the important predictors for MVI. Considering the increased incidence of vascular invasion on pathology as tumor size increased [57], a larger tumor size would be associated with MVI. Because most individual studies compared the mean tumor size between HCCs with MVI and those without MVI by continuous variables, or used a different cutoff value of tumor size, 
the number of studies available to calculate DOR with the same cutoff value $(>5 \mathrm{~cm})$ was 4 in this meta-analysis. When we compared our findings with those of individual studies which compared tumor size between HCCs with MVI and those without MVI by continuous variables, our meta-analysis was concordant with 8 studies [2, $11,14,17,31,41-43]$, but nonconcordant with 11 studies $[9,11,18,19,22,34-36,45-47]$. However, a large tumor size $(>5 \mathrm{~cm})$ as a predictor for MVI would not be useful in the selection of treatment modalities (surgical resection vs. RFA) because RFA is not usually considered for HCC of $>5 \mathrm{~cm}$. Therefore, other MRI features could be more useful for selecting the optimal curative treatment modality (surgical resection vs. RFA).

Of the 7 available studies evaluating diffusion restriction, 3 studies reported that diffusion restriction was a significant predictor of MVI $[28,32,40]$, but 4 studies did not show a statistically significant association $[16,23,34$, 39]. In subgroup analysis, diffusion restriction was significantly associated with MVI on 1.5-T MRI, but not significantly associated with it on 3.0-T MRI. Given the association between the ADC values and histologically differentiation grade of HCC [32], one could expect diffusion restriction (lower ADC values) to be a predictor of MVI. However, because ADC values may differ according to MRI coil systems, vendors, and field strength [58], and the fact that there was variability in the definition of diffusion restriction in each individual study, the reported results may vary across studies, and these heterogeneities could have reduced the statistical significance. In addition, the small number of studies (i.e., only 3 studies using 3.0-T MRI) might have limited the ability to show a statistically significant association.

In addition to the performance of individual MRI features for predicting MVI, the diagnostic performance of combined features can also be useful $[10,59]$. Compared with individual findings in this meta-analysis, that is, peritumoral hypointensity on HBP, a combination of MRI features had higher sensitivity (52.4 vs. $44.2 \%)$ and similar specificity (92.5 vs. $91.1 \%$ ) [10]. Although the diagnostic performance of combined feature models could not be evaluated in this meta-analysis because of limitations in statistical methods, the 7 significant MRI features identified in this meta-analysis should provide guidance for further such studies.

This study has some limitations. Notably, substantial study heterogeneity was noted across the included studies. Because most studies $(91.7 \%, 33 / 36)$ were retrospective, study heterogeneity across them might be inevitable. Although we investigated the causes for the heterogeneity by performing meta-regression analysis, the results of meta-regression analysis cannot be sufficient to fully explain the study heterogeneity. Therefore, general applicability of the results may warrant caution due to high study heterogeneity among MRI features. Second, there was variability in the definition of MRI features. However, to minimize this variability, we carefully subsumed under a single MRI feature with a consideration of the original definition of the MRI feature in each individual study.

\section{Conclusion}

Seven MRI features including larger tumor size $(>5$ $\mathrm{cm})$, rim arterial enhancement, arterial peritumoral enhancement, peritumoral hypointensity on HBP, nonsmooth margin, multifocality, and hypointensity on T1WI were significant predictors for MVI of HCC. These MRI features predictive of MVI can be useful in the management of HCC.

\section{Statement of Ethics}

Institutional Review Board approval was not required due to study design, which was a systematic review and meta-analysis.

\section{Conflict of Interest Statement}

S.H. Choi is an advisory board member of Bayer Healthcare and receives research funding from Bayer Healthcare.

\section{Funding Sources}

This work was supported by the National Research Foundation of Korea (NRF) grant funded by the Korea government (MSIT) (Grant No. NRF-2019R1G1A1099743).

\section{Author Contributions}

S.B. Hong and S.H. Choi contributed to the study concept and design. S.B. Hong and K.W. Kim acquired the data. S.H. Choi, S.B. Hong, S.Y. Kim, and S.S. Lee analyzed and interpreted the data. S.B. Hong, S.H. Choi, and J.H. Shim drafted the manuscript. S.H. Choi performed statistical analysis. S.H. Choi, J.H. Shim, J.H. Byun, S.H. Park, S. Kim, and N.K. Lee made critical revisions to the manuscript. S.H. Choi supervised the study. 


\section{References}

1 Mazzaferro V, Llovet JM, Miceli R, Bhoori S, Schiavo M, Mariani L, et al. Predicting survival after liver transplantation in patients with hepatocellular carcinoma beyond the Milan criteria: a retrospective, exploratory analysis. Lancet Oncol. 2009 Jan;10(1):35-43.

2 Lee S, Kang TW, Song KD, Lee MW, Rhim H, Lim HK, et al. Effect of microvascular invasion risk on early recurrence of hepatocellular carcinoma after surgery and radiofrequency ablation. Ann Surg. 2019 Apr 30.

3 Zhang X, Li J, Shen F, Lau WY. Significance of presence of microvascular invasion in specimens obtained after surgical treatment of hepatocellular carcinoma. J Gastroenterol Hepatol. 2018 Feb;33(2):347-54.

4 Nuta J, Shingaki N, Ida Y, Shimizu R, Hayami $\mathrm{S}$, Ueno $\mathrm{M}$, et al. Irregular defects in hepatocellular carcinomas during the Kupffer phase of contrast-enhanced ultrasonography with perfluorobutane microbubbles: pathological features and metastatic recurrence after surgical resection. Ultrasound Med Biol. 2017 Sep; 43(9):1829-36

5 Chou CT, Chen RC, Lee CW, Ko CJ, Wu HK, Chen YL. Prediction of microvascular invasion of hepatocellular carcinoma by pre-operative CT imaging. Br J Radiol. 2012 Jun; 85(1014):778-83.

6 Mori Y, Tamai H, Shingaki N, Moribata K, Deguchi H, Ueda K, et al. Signal intensity of small hepatocellular carcinoma on apparent diffusion coefficient mapping and outcome after radiofrequency ablation. Hepatol Res. 2015 Jan;45(1):75-87.

7 Kim KA, Kim MJ, Jeon HM, Kim KS, Choi JS, Ahn SH, et al. Prediction of microvascular invasion of hepatocellular carcinoma: usefulness of peritumoral hypointensity seen on gadoxetate disodium-enhanced hepatobiliary phase images. J Magn Reson Imaging. 2012 Mar;35(3):629-34.

8 Kim AY, Sinn DH, Jeong WK, Kim YK, Kang TW, Ha SY, et al. Hepatobiliary MRI as novel selection criteria in liver transplantation for hepatocellular carcinoma. J Hepatol. 2018 Jun;68(6):1144-52.

9 Ariizumi S, Kitagawa K, Kotera Y, Takahashi Y, Katagiri S, Kuwatsuru R, et al. A nonsmooth tumor margin in the hepatobiliary phase of gadoxetic acid disodium (Gd-EOBDTPA)-enhanced magnetic resonance imaging predicts microscopic portal vein invasion, intrahepatic metastasis, and early recurrence after hepatectomy in patients with hepatocellular carcinoma. J Hepatobiliary Pancreat Sci. 2011 Jul;18(4):575-85.

10 Lee S, Kim SH, Lee JE, Sinn DH, Park CK. Preoperative gadoxetic acid-enhanced MRI for predicting microvascular invasion in patients with single hepatocellular carcinoma. J Hepatol. 2017 Sep;67(3):526-34.
11 Huang M, Liao B, Xu P, Cai H, Huang K, Dong Z, et al. Prediction of microvascular invasion in hepatocellular carcinoma: preoperative Gd-EOB-DTPA-dynamic enhanced MRI and histopathological correlation. Contrast Media Mol Imaging. 2018;2018:9674565.

12 Ryu T, Takami Y, Wada Y, Tateishi M, Hara $\mathrm{T}$, Yoshitomi M, et al. A clinical scoring system for predicting microvascular invasion in patients with hepatocellular carcinoma within the Milan criteria. J Gastrointest Surg. 2019 Apr;23(4):779-87.

13 Rhee H, An C, Kim HY, Yoo JE, Park YN, Kim MJ. Hepatocellular carcinoma with irregular rim-like arterial phase hyperenhancement: more aggressive pathologic features. Liver Cancer. 2019 Feb;8(1):24-40.

14 Kim H, Park MS, Choi JY, Park YN, Kim MJ, Kim KS, et al. Can microvessel invasion of hepatocellular carcinoma be predicted by pre-operative MRI? Eur Radiol. 2009 Jul;19(7):1744-51.

15 Witjes CD, Willemssen FE, Verheij J, van der Veer SJ, Hansen BE, Verhoef C, et al. Histological differentiation grade and microvascular invasion of hepatocellular carcinoma predicted by dynamic contrast-enhanced MRI. J Magn Reson Imaging. 2012 Sep;36(3):641-7.

16 Ahn SJ, Kim JH, Park SJ, Kim ST, Han JK. Hepatocellular carcinoma: preoperative gadoxetic acid-enhanced MR imaging can predict early recurrence after curative resection using image features and texture analysis. Abdom Radiol. 2019 Feb;44(2):539-48.

17 Suh YJ, Kim MJ, Choi JY, Park MS, Kim KW . Preoperative prediction of the microvascular invasion of hepatocellular carcinoma with diffusion-weighted imaging. Liver Transpl. 2012 Oct;18(10):1171-8.

18 Xu P, Zeng M, Liu K, Shan Y, Xu C, Lin J. Microvascular invasion in small hepatocellular carcinoma: is it predictable with preoperative diffusion-weighted imaging? J Gastroenterol Hepatol. 2014 Feb;29(2):330-6.

19 Zhao J, Li X, Zhang K, Yin X, Meng X, Han L, et al. Prediction of microvascular invasion of hepatocellular carcinoma with preoperative diffusion-weighted imaging: a comparison of mean and minimum apparent diffusion coefficient values. Medicine. 2017 Aug;96(33):e7754.

20 Zhao W, Liu W, Liu H, Yi X, Hou J, Pei Y, et al. Preoperative prediction of microvascular invasion of hepatocellular carcinoma with IVIM diffusion-weighted MR imaging and Gd-EOB-DTPA-enhanced MR imaging. PLoS One. 2018 May 17;13(5):e0197488.

21 Lee S, Kim KW, Jeong WK, Kim MJ, Choi $\mathrm{GH}$, Choi JS, et al. Gadoxetic acid-enhanced MRI as a predictor of recurrence of HCC after liver transplantation. Eur Radiol. 2020 Feb; 30(2):987-95.

22 Chandarana $\mathrm{H}$, Robinson E, Hajdu CH, Drozhinin L, Babb JS, Taouli B. Microvascular invasion in hepatocellular carcinoma: is it predictable with pretransplant MRI? AJR Am J Roentgenol. 2011 May;196(5):1083-9.
23 Granata V, Fusco R, Setola SV, Picone C, Vallone $\mathrm{P}$, Belli A, et al. Microvascular invasion and grading in hepatocellular carcinoma: correlation with major and ancillary features according to LIRADS. Abdom Radiol. 2019 Aug;44(8):2788-800.

24 Reitsma JB, Glas AS, Rutjes AW, Scholten RJ, Bossuyt PM, Zwinderman AH. Bivariate analysis of sensitivity and specificity produces informative summary measures in diagnostic reviews. J Clin Epidemiol. 2005 Oct;58(10): 982-90.

25 Higgins JP, Thompson SG, Deeks JJ, Altman DG. Measuring inconsistency in meta-analyses. BMJ. 2003 Sep;327(7414):557-60.

26 Deville WL, Buntinx F, Bouter LM, Montori VM, de Vet HC, van der Windt DA, et al. Conducting systematic reviews of diagnostic studies: didactic guidelines. BMC Med Res Methodol. 2002 Jul;2:9.

27 Kim JY, Kim MJ, Kim KA, Jeong HT, Park YN. Hyperintense HCC on hepatobiliary phase images of gadoxetic acid-enhanced MRI: correlation with clinical and pathological features. Eur J Radiol. 2012 Dec;81(12): 3877-82.

28 Kim MJ, Lee M, Choi JY, Park YN. Imaging features of small hepatocellular carcinomas with microvascular invasion on gadoxetic acid-enhanced MR imaging. Eur J Radiol. 2012 Oct;81(10):2507-12.

29 Nishie A, Asayama Y, Ishigami K, Kakihara D, Nakayama T, Ushijima Y, et al. Clinicopathological significance of the peritumoral decreased uptake area of gadolinium ethoxybenzyl diethylenetriamine pentaacetic acid in hepatocellular carcinoma. J Gastroenterol Hepatol. 2014 Mar;29(3):561-7.

30 Ahn SY, Lee JM, Joo I, Lee ES, Lee SJ, Cheon GJ, et al. Prediction of microvascular invasion of hepatocellular carcinoma using gadoxetic acid-enhanced MR and (18)F-FDG PET/CT. Abdom Imaging. 2015 Apr;40(4):843-51.

31 Min JH, Kim YK, Lim S, Jeong WK, Choi D, Lee WJ. Prediction of microvascular invasion of hepatocellular carcinomas with gadoxetic acid-enhanced MR imaging: impact of intratumoral fat detected on chemical-shift images. Eur J Radiol. 2015 Jun;84(6):1036-43.

32 Mori Y, Tamai H, Shingaki N, Hayami S, Ueno M, Maeda Y, et al. Hypointense hepatocellular carcinomas on apparent diffusion coefficient mapping: pathological features and metastatic recurrence after hepatectomy. Hepatol Res. 2016 Jun;46(7):634-41.

33 Shin SK, Kim YS, Shim YS, Choi SJ, Park SH, Jung DH, et al. Peritumoral decreased uptake area of gadoxetic acid enhanced magnetic resonance imaging and tumor recurrence after surgical resection in hepatocellular carcinoma: A STROBE-compliant article. Medicine. 2017 Aug;96(33):e7761. 
34 Yang C, Wang H, Sheng R, Ji Y, Rao S, Zeng $\mathrm{M}$. Microvascular invasion in hepatocellular carcinoma: is it predictable with a new, preoperative application of diffusion-weighted imaging? Clin Imaging. 2017 Jan- Feb;41: 101-5.

35 Zhou XM, Wang G, Gao YX, Yu L, Li W, Jiao $\mathrm{LL}$, et al. Predict microvascular invasion in hepatocellular carcinoma by dynamic contrast-enhanced magnetic resonance imaging in patients with hepatitis B virus. Int J Clin Exp Med. 2017;10(8):11728-38.

36 Yang C, Wang H, Tang Y, Rao S, Sheng R, Ji $\mathrm{Y}$, et al. ADC similarity predicts microvascular invasion of bifocal hepatocellular carcinoma. Abdom Radiol. 2018 Sep;43(9):2295302.

37 Yoneda N, Matsui O, Kitao A, Komori T, Kozaka K, Ikeda H, et al. Peri-tumoral hyperintensity on hepatobiliary phase of gadoxetic acid-enhanced MRI in hepatocellular carcinomas: correlation with peri-tumoral hyperplasia and its pathological features. Abdom Radiol. 2018 Aug;43(8):2103-12.

38 Feng ST, Jia Y, Liao B, Huang B, Zhou Q, Li $X$, et al. Preoperative prediction of microvascular invasion in hepatocellular cancer: a radiomics model using Gd-EOB-DTPA-enhanced MRI. Eur Radiol. 2019 Sep;29(9): 4648-59.

39 Lee S, Kim SH, Hwang JA, Lee JE, Ha SY. Preoperative ADC predicts early recurrence of HCC after curative resection. Eur Radiol. 2019 Feb;29(2):1003-12.

40 Server S, Sabet S, Yaghouti K, Namal E, Inan $\mathrm{N}$, Tokat $\mathrm{Y}$. Value of imaging findings in the prediction of microvascular invasion in hepatocellular carcinoma. Transplant Proc. 2019 Sep;51(7):2403-7.

41 Yang L, Gu D, Wei J, Yang C, Rao S, Wang W, et al. A radiomics nomogram for preoperative prediction of microvascular invasion in hepatocellular carcinoma. Liver Cancer. 2019 Oct; 8(5):373-86.

42 Zhang R, Xu L, Wen X, Zhang J, Yang P, Zhang L, et al. A nomogram based on bi-regional radiomics features from multimodal magnetic resonance imaging for preoperative prediction of microvascular invasion in hepatocellular carcinoma. Quant Imaging Med Surg. 2019 Sep;9(9):1503-15.

43 Zhu YJ, Feng B, Wang S, Wang LM, Wu JF, $\mathrm{Ma} \mathrm{XH}$, et al. Model-based three-dimensional texture analysis of contrast-enhanced magnetic resonance imaging as a potential tool for preoperative prediction of microvascular invasion in hepatocellular carcinoma. Oncol Lett. 2019 Jul;18(1):720-32.

44 Sun SW, Liu QP, Xu X, Zhu FP, Zhang YD, Liu XS. Direct comparison of four presurgical stratifying schemes for prediction of microvascular invasion in hepatocellular carcinoma by gadoxetic acid-enhanced MRI. J Magn Reson Imaging. 2020 Aug;52(2):433-47.

45 Jhaveri KS, Cleary SP, Fischer S, Haider MA, Pargoankar V, Khalidi K, et al. Blood oxygen level-dependent liver MRI: can it predict microvascular invasion in HCC? J Magn Reson Imaging. 2013 Mar;37(3):692-9.

46 Wang WT, Yang L, Yang ZX, Hu XX, Ding Y, Yan X, et al. Assessment of microvascular invasion of hepatocellular carcinoma with diffusion kurtosis imaging. Radiology. $2018 \mathrm{Feb}$; 286(2):571-80.

47 Wei Y, Huang Z, Tang H, Deng L, Yuan Y, Li $\mathrm{J}$, et al. IVIM improves preoperative assessment of microvascular invasion in HCC. Eur Radiol. 2019 Oct;29(10):5403-14.

48 Halligan S, Altman DG. Evidence-based practice in radiology: steps 3 and 4--appraise and apply systematic reviews and meta-analyses. Radiology. 2007 Apr;243(1):13-27.

49 Choi JY, Lee JM, Sirlin CB. CT and MR imaging diagnosis and staging of hepatocellular carcinoma: part II. Extracellular agents, hepatobiliary agents, and ancillary imaging features. Radiology. 2014 Oct;273(1):30-50.

50 Matsui O, Kobayashi S, Sanada J, Kouda W, Ryu Y, Kozaka K, et al. Hepatocelluar nodules in liver cirrhosis: hemodynamic evaluation (angiography-assisted CT) with special reference to multi-step hepatocarcinogenesis. Abdom Imaging. 2011 Jun;36(3):264-72.

51 Nakashima Y, Nakashima O, Tanaka M, Okuda K, Nakashima M, Kojiro M. Portal vein invasion and intrahepatic micrometastasis in small hepatocellular carcinoma by gross type. Hepatol Res. 2003 Jun;26(2):142-7.

52 Shimada M, Rikimaru T, Hamatsu T, Yamashita Y, Terashi T, Taguchi K, et al. The role of macroscopic classification in nodulartype hepatocellular carcinoma. Am J Surg. 2001 Aug; 182(2):177-82.

53 An C, Kim DW, Park YN, Chung YE, Rhee H, Kim MJ. Single hepatocellular carcinoma: preoperative MR imaging to predict early recurrence after curative resection. Radiology. 2015 Aug;276(2):433-43.

54 Esnaola NF, Lauwers GY, Mirza NQ, Nagorney DM, Doherty D, Ikai I, et al. Predictors of microvascular invasion in patients with hepatocellular carcinoma who are candidates for orthotopic liver transplantation. J Gastrointest Surg. 2002 Mar-Apr;6(2):224-32

55 Enomoto S, Tamai H, Shingaki N, Mori Y, Moribata K, Shiraki T, et al. Assessment of hepatocellular carcinomas using conventional magnetic resonance imaging correlated with histological differentiation and a serum marker of poor prognosis. Hepatol Int. 2011 Jun;5(2):730-7.

56 Pawlik TM, Delman KA, Vauthey JN, Nagorney DM, Ng IO, Ikai I, et al. Tumor size predicts vascular invasion and histologic grade: implications for selection of surgical treatment for hepatocellular carcinoma. Liver Transpl. 2005 Sep;11(9):1086-92.

57 Eguchi S, Takatsuki M, Hidaka M, Soyama A, Tomonaga T, Muraoka I, et al. Predictor for histological microvascular invasion of hepatocellular carcinoma: a lesson from 229 consecutive cases of curative liver resection. World J Surg. 2010 May;34(5):1034-8.

58 Sasaki M, Yamada K, Watanabe Y, Matsui M, Ida M, Fujiwara S, et al. Variability in absolute apparent diffusion coefficient values across different platforms may be substantial: a multivendor, multi-institutional comparison study. Radiology. 2008 Nov;249(2):624-30.

59 Renzulli M, Brocchi S, Cucchetti A, Mazzotti F, Mosconi C, Sportoletti C, et al. Can current preoperative imaging be used to detect microvascular invasion of hepatocellular carcinoma? Radiology. 2016 May;279(2):432-42. 